Investigaciones geográficas, $n .^{\circ} 45$ (2008) pp. 203-227 ISSN: 0213-4691
Instituto Universitario de Geografía Universidad de Alicante

\title{
RIESGO DE INUNDACIÓN Y ORDENACIÓN TERRITORIAL EN EL ÁREA LITORAL SUR DE LA MARINA BAIXA (ALICANTE)
}

\author{
José Marcos Ortega Álvarez \\ Departamento de Ingeniería de la Construcción, \\ Obras Públicas e Infraestructura Urbana \\ Universidad de Alicante
}

\section{Introducción}

Las inundaciones son los desastres naturales con mayor repercusión socioeconómica, tanto a escala mundial como en lo que se refiere a nuestro país. Además, si hablamos del coste en vidas humanas, éstas suponen un goteo anual continuo, concentrado en eventos que han causado una profunda alarma social. En el caso del sureste peninsular, los episodios de lluvia torrencial que precipitan en régimen tormentoso son los sucesos atmosféricos que causan daños más cuantiosos. Estas precipitaciones producen la crecida de ramblas y barrancos, que trae consigo la destrucción de infraestructuras, anegación de campos e inundación de tramas urbanas.

Ante esta situación, es necesario adoptar una serie de medidas para tratar de minimizar el riesgo relacionado con las inundaciones y paliar sus consecuencias. Entre estas medidas están las predictivas, las preventivas y las correctoras. Todas ellas precisan como paso previo y fundamental la realización de un estudio de riesgo de inundaciones, dentro de los cuales la cartografía de riesgo es un componente básico, ya que permite representar eficazmente la distribución espacial de los diferentes elementos de la severidad (profundidad, velocidad, carga sólida...), la dimensión espacio-temporal (zonas inundables, tiempos característicos...) y la frecuencia (períodos de retorno, probabilidad de excedencia...) del fenómeno de la inundación. 
Figura 1. Localización de la zona de estudio

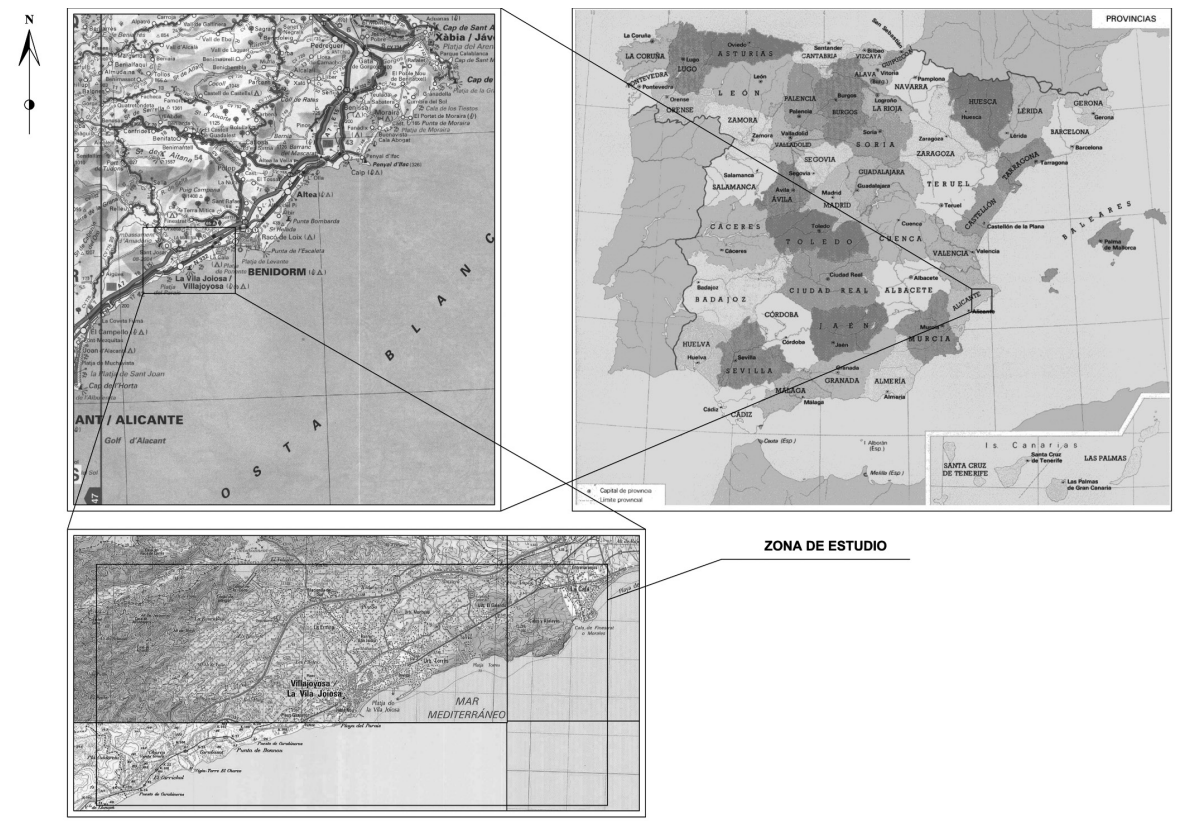

En este trabajo se presenta un método de análisis de riesgo de inundación y se aplica a una zona concreta, que ha sido el área litoral meridional de la comarca de la Marina Baixa (provincia de Alicante). Exactamente, el análisis del riesgo de inundación se ha realizado en los tramos finales de los principales barrancos que desembocan en el litoral de los términos municipales de Villajoyosa y Finestrat.

La metodología, los pasos y herramientas que se han seguido son un compendio de los diferentes propuestos en nuestro país para este tipo de estudios. Se ha intentado establecer un denominador común de todos ellos, haciendo especial hincapié en el caso particular de la Comunidad Valenciana. A parte del estudio hidráulico e hidrológico, también se han inventariado las ocupaciones antrópicas de los cauces y se ha expuesto el tratamiento que realiza la documentación oficial del riesgo de inundación en el área analizada.

En lo referente a las características geográficas de la zona, la localidad de Villajoyosa ${ }^{1}$ está situada a una distancia de $32 \mathrm{~km}$ de Alicante, siendo su

1. Los datos de Villajoyosa y Finestrat se han obtenido de su ficha municipal existente en la página web del Instituto Valenciano de Estadística (IVE), cuya dirección es <http:// www.ive.es>. 
altitud de $27 \mathrm{~m}$ y la superficie del término municipal de $59,3 \mathrm{~km}^{2}$. La población en 2005 era de 27983 habitantes, presentando una densidad de población $472,3 \mathrm{hab} / \mathrm{km}^{2}$. Por otro lado, la localidad de Finestrat se halla a unos $40 \mathrm{~km}$ de Alicante, siendo la superficie del término municipal de $42,3 \mathrm{~km}^{2}$. Consta de dos núcleos de población importantes, que son la propia localidad de Finestrat, ubicada al pie del Puig Campana, y la Cala de Finestrat situada en la costa. La población censada en el término municipal es de 3797 habitantes, presentando una densidad de población de $89,9 \mathrm{hab} / \mathrm{km}^{2}$. La cifra de habitantes censados es engañosa, ya que en la Cala de Finestrat existen muchos edificios de apartamentos y segundas residencias, por lo que sus propietarios sólo las habitan durante los períodos vacacionales, especialmente verano.

El crecimiento de la población durante los últimos siete años en la zona estudiada ha sido considerable. Concretamente, en Villajoyosa la población ha aumentado en más de 4000 habitantes, representando un incremento del 18\%, mientras que en Finestrat el crecimiento ha sido de más de 1500 habitantes, lo que ha supuesto un incremento del $75 \%$.

Respecto al contexto geológico ${ }^{2}$, las litologías presentes en la totalidad de cuencas estudiadas están compuestas principalmente por sucesiones de margas arcillosas, biocalcarenitas y niveles calcáreos bastante fosilíferos, de edad paleógena y miocena (constituyen el flysch existente entre Campello y Villajoyosa). La zona de la huerta de Villajoyosa está formada por depósitos del Cuaternario, constituidos por gravas, arenas y limos arcillosos de tonalidad amarillenta. El lecho de los cauces está relleno de gravas, arenas y arcillas. Mención a parte tiene la cuenca del río Torres, que abarca una extensión mucho mayor, aflorando en su parte alta arcillas y yesos de edad triásica. También destaca la presencia de calizas del Jurásico y del Cretácico, a medida que nos acercamos a la zona de nacimiento del cauce principal.

En este tipo de estudios tampoco hay que olvidar la importancia de las inundaciones acaecidas en tiempos pasados, que sin duda son de gran utilidad para conocer las zonas más proclives a ser anegadas por las aguas de avenida y para comprobar que los modelos empleados en la elaboración de la cartografía de riesgo de inundación dan resultados coherentes. En la zona de estudio, las principales inundaciones se han producido debido a avenidas del río Amadorio (popularmente conocidas como «Rovina»), afectando a las viviendas situadas en los alrededores de la desembocadura, y a las avenidas de la cuenca del Barranco Refoio que han producido daños muy importantes en el núcleo urbano (a éstas se las conoce como «Refoios»). En menor medida, también

2. Memoria de la hoja 847 «Villajoyosa» del Mapa geológico de España a escala 1:50 000. 
se ha visto afectada por inundaciones la zona de la Cala del Torres, donde desemboca el río homónimo y el barranco Castilla, especialmente durante las décadas en las que estuvieron situados en ella dos camping.

Sobre las avenidas del Río Amadorio, es importante mencionar que eran bastantes frecuentes antes de la construcción del pantano del Amadorio, inaugurado el 17 de junio de 1956. Desde entonces únicamente se han dado inundaciones en la zona de la desembocadura en las contadas ocasiones en que se ha tenido que desaguar a través de los aliviaderos de la presa. Las fechas de las inundaciones más importantes acaecidas durante el siglo XX en la zona de estudio son las siguientes:

Inundaciones de 24 y 25 de septiembre de 1931.

Inundaciones entre el 31 de octubre y el 2 de noviembre de 1934.

Inundaciones entre el 8 y 11 de octubre de 1943.

Riada entre el 15 y 18 de octubre de 1950.

Inundaciones entre el 5 y 7 de Octubre de 1971.

Inundaciones de 20 y 21 de Octubre de 1982.

Inundaciones de 4 y 5 de Noviembre de 1987.

\section{Método de trabajo y cartografía de riesgo}

Para el trazado de las divisorias entre las cuencas y de los principales cauces que las componen, se ha empleado como base el mapa topográfico a escala 1:10000 editado por el Institut Cartogràfic Valencià.

La caracterización de las cuencas ha consistido básicamente en calcular una serie de parámetros (véase la tabla 1) agrupados en parámetros generales de la cuenca, parámetros de forma, características del relieve, características de la red de drenaje y características del cauce principal.

El umbral de escorrentía es la altura mínima de lluvia necesaria para que se produzca escorrentía superficial. Para su cálculo se ha procedido a seguir las directrices indicadas por la Instrucción de carreteras 5.2-IC: «Drenaje superficial». Según ella, el umbral de escorrentía se halla en función de cuatro parámetros, que son el uso del suelo, tipo de suelo, pendiente y características hidrológicas.

En este estudio, las precipitaciones máximas diarias en las cuencas para los períodos de retorno de 25, 100 y 500 años se han determinado de dos formas diferentes.

La primera de ellas ha consistido en localizar las estaciones pluviométricas próximas a la zona de estudio que tuviesen series de registros lo suficientemente largas para la aplicación de un método estadístico. Las estaciones empleadas han sido las de Relleu (serie 1952-2006) y Sella (serie 1965-1997). 
Tabla 1. Parámetros para la caracterización de las cuencas

\begin{tabular}{|c|c|}
\hline \multirow[t]{2}{*}{ Parámetros generales de la cuenca } & Superficie \\
\hline & Perímetro \\
\hline \multirow[t]{5}{*}{ Parámetros de forma } & Coeficiente de compacidad \\
\hline & Coeficiente de forma \\
\hline & Factor de forma \\
\hline & Radio de elongación \\
\hline & Radio de circularidad \\
\hline \multirow[t]{8}{*}{ Características del relieve } & Curva de frecuencias \\
\hline & Curva hipsométrica \\
\hline & Altitud media de la cuenca \\
\hline & Rectángulo equivalente \\
\hline & Pendiente media de la cuenca \\
\hline & Índice de pendiente \\
\hline & Índice de pendiente relativo \\
\hline & Coeficiente orográfico \\
\hline \multirow[t]{7}{*}{ Red de drenaje } & Longitudes totales por órdenes de cauce \\
\hline & Relación de bifurcación \\
\hline & Densidad de drenaje \\
\hline & Canal de alimentación \\
\hline & Densidad hidrográfica \\
\hline & Alejamiento medio \\
\hline & Coeficiente de torrencialidad \\
\hline \multirow[t]{3}{*}{ Cauce principal } & Perfil longitudinal \\
\hline & Pendiente media del cauce principal \\
\hline & Coeficiente de sinuosidad \\
\hline
\end{tabular}

Para la obtención de las precipitaciones en los períodos de retorno considerados partiendo de los registros, se ha desarrollado un modelo de análisis de frecuencias utilizando la distribución de valores extremos tipo I (Gumbel). Además, para determinar si el ajuste realizado es bueno desde el punto de vista estadístico se ha empleado el test de bondad del ajuste de KolmogorovSmirnov. Finalmente, una vez halladas las precipitaciones en ambas estaciones, se han determinado las precipitaciones correspondientes a cada cuenca empleando los polígonos de Thiessen.

El segundo método ha consistido en determinar las precipitaciones máximas mediante el mapa de Máximas lluvias diarias en la España peninsular, editado por la Dirección General de Carreteras del Ministerio de Fomento. La finalidad de este documento es facilitar el cálculo de los caudales a desaguar 
por los pequeños cauces existentes en las obras de carreteras, supliendo así la ausencia de aforos en los mismos. La función de distribución que emplea es la SQRT-ET max y para tener una mayor operatividad, la publicación incluye una cartografía de España en la que se representan las isolíneas de un coeficiente de variación $\left(C_{v}\right)$ y las del valor medio $(P)$ de la máxima precipitación diaria anual.

Para cada una de las cuencas se han comparado los valores de precipitación obtenidos por ambos métodos para cada período de retorno, y se ha seleccionado el mayor de ambos, que a su vez es el más desfavorable, para realizar los posteriores cálculos. A título anecdótico, hay que indicar que las precipitaciones halladas mediante ambos métodos diferían muy poco.

Para el cálculo de los caudales máximos se ha empleado el método racional, siguiendo la formulación propuesta por Témez en 1991. En este método no es necesario conocer la duración de la precipitación y es aconsejado su empleo por parte de la Instrucción 5.2-IC. Se ha descartado el empleo de hidrogramas al carecer de suficientes registros de duración de precipitaciones.

La elaboración de la cartografía de riesgo de inundación se ha realizado siguiendo las directrices del «Plan de acción territorial de carácter sectorial sobre prevención del riesgo de inundación de la Comunidad Valenciana (PATRICOVA)», que establece 6 niveles de riesgo:

Riesgo 1.- Frecuencia menor de 25 años con calado superior a $0,8 \mathrm{~m}$.

Riesgo 2.- Frecuencia entre 25 y 100 años con calado superior a $0,8 \mathrm{~m}$.

Riesgo 3.- Frecuencia menor de 25 años con calado inferior a $0,8 \mathrm{~m}$.

Riesgo 4.- Frecuencia entre 25 y 100 años con calado inferior a 0,8 m.

Riesgo 5.- Frecuencia entre 100 y 500 años con calado superior a $0,8 \mathrm{~m}$.

Riesgo 6.- Frecuencia entre 100 y 500 años con calado inferior a $0,8 \mathrm{~m}$.

La modelización hidráulica se ha efectuado mediante el programa HECRAS $^{3}$ creado por el US Army Corps of Engineers (Cuerpo de Ingenieros del Ejército de Estados Unidos de América). La cartografía de riesgo de inundación se ha efectuado en el tramo final del cauce principal de cada cuenca, cuya longitud según los casos, está comprendida entre los 500 y 1000 m teniendo como punto final la desembocadura. La base topográfica para el trabajo ha sido la cartografía contenida en el Plan General de Ordenación Urbana (PGOU) de Villajoyosa a escala 1:2000, con equidistancia entre curvas de nivel de $2 \mathrm{~m}$. La representación de las áreas inundables sobre esta cartografía ha sido realizada con el programa AutoCAD Map de la empresa Autodesk. Para transferir

3. Siglas de «Hydrologic Engineering Center - River Analysis System». Se puede descargar gratuitamente en la página web <http://www.hec.usace.army.mil/software/hec-ras/>. 
los resultados obtenidos en HEC-RAS a AutoCAD Map, se ha empleado la aplicación informática Lámina .

A lo largo de todo el estudio se han analizado un total de 222 secciones transversales, de las cuales 30 han sido medidas directamente en el campo, empleando material topográfico como el nivel automático, la mira estadimétrica, el GPS y la cinta métrica. La separación entre secciones ha sido $25 \mathrm{~m}$. Los valores del coeficiente de rugosidad o de Manning en cada una de las secciones se han estimado en el campo a partir de clasificación publicada por la Organización de las Naciones Unidas para la Agricultura y la Alimentación (FAO).

Hay que indicar que la modelización hidráulica mediante el programa HEC-RAS se ha realizado con flujo estacionario, tomando como condición de contorno la de calado normal. En esta condición se introduce la pendiente de la línea de energía, que como se desconoce, se ha aproximado su valor a la pendiente del cauce en el tramo estudiado.

El análisis de la ocupación antrópica de cauces se ha realizado directamente en el campo. Ha consistido en recorrer los cauces de los barrancos estudiados, observando las ocupaciones existentes y las intersecciones con las obras lineales, tratando de evaluar in situ si éstas son adecuadas o no y si podrían haberse resuelto de una forma mejor.

En cada uno de los barrancos también se ha estudiado el tratamiento que realiza la documentación oficial del riesgo de inundación existente en cada uno de ellos. Los documentos analizados han sido el PATRICOvA, el Plan General de Ordenación Urbana de Villajoyosa y las normas subsidiarias vigentes todavía en Finestrat para regular el planeamiento urbanístico en el momento de la realización de este estudio.

Finalmente, se han recopilado una serie de datos complementarios fundamentalmente de carácter histórico. Para ello, se han compilado fotografías antiguas sobre todo de episodios de inundación anteriores, se ha buscado información en diversos libros y periódicos y se han efectuado encuestas de campo a personas mayores.

4. Esta aplicación ha sido creada por el profesor Allen Bateman Pinzón, perteneciente al Grupo de Investigación en Transporte de Sedimentos (GITS) de la Sección de Ingeniería Hidráulica e Hidrológica adscrita a la E.T.S. de Ingeniería de Caminos, Canales y Puertos de la Universidad Politécnica de Cataluña. Se puede descargar gratuitamente en la web $<$ http://www.gits.ws/03software/software.php>. 


\section{Análisis del riesgo de inundación en el área litoral sur de la Marina} Baixa

Las cuencas en las que se ha analizado el riesgo de inundación son las siguientes ${ }^{5}$ :

Barranco Carrichal.

Barranco Aigüeta.

Barranco Alfondó.

Río Amadorio.

Barranco Refoio.

Río Torres.

Barranco Castilla.

Barranco de la Cala de Finestrat.

Los datos más importantes de cada una de ellas se pueden consultar en la tabla 2.

\subsection{Análisis del riesgo de inundación en el tramo final del barranco Carrichal}

Este barranco nace en la partida Vall-llongues del término municipal de Orcheta. Su cauce principal en el curso medio y bajo constituye el límite entre los términos municipales de El Campello y Villajoyosa. Este barranco también recibe el nombre en valenciano de barranc del Carritxar.

Los puntos más problemáticos son las intersecciones con la autopista AP7 y con la carretera N-332. La primera de ellas se ha resuelto mediante una canalización prefabricada de hormigón con sección semicircular. Hay que indicar que también se aprovecha para que un camino cruce la autopista. Junto a esta obra de fábrica es difícil distinguir el cauce, dada la gran cantidad de tierra que se movilizó durante la construcción de la autopista en este punto, dando lugar a un terraplén de gran altura. No obstante, la canalización es suficiente para desaguar los caudales calculados en este punto. El cruce del barranco con el trazado de la carretera N-332 se lleva a cabo mediante un puente de una única luz, con sección en arco de medio punto elevado por hastiales, dándole un aspecto alto y estrecho. La vegetación en este lugar es más abundante, obstruyendo la entrada al puente, hecho que se agrava por el vertido de escombros en ambas márgenes. La sección parece suficiente, aunque sería necesaria una limpieza de la vegetación y escombros en sus inmediaciones para mejorar su capacidad de desagüe.

5. Están ordenadas de suroeste a noreste, es decir, empezando desde el límite municipal entre El Campello y Villajoyosa y terminando en el límite entre los términos de Finestrat y Benidorm. 


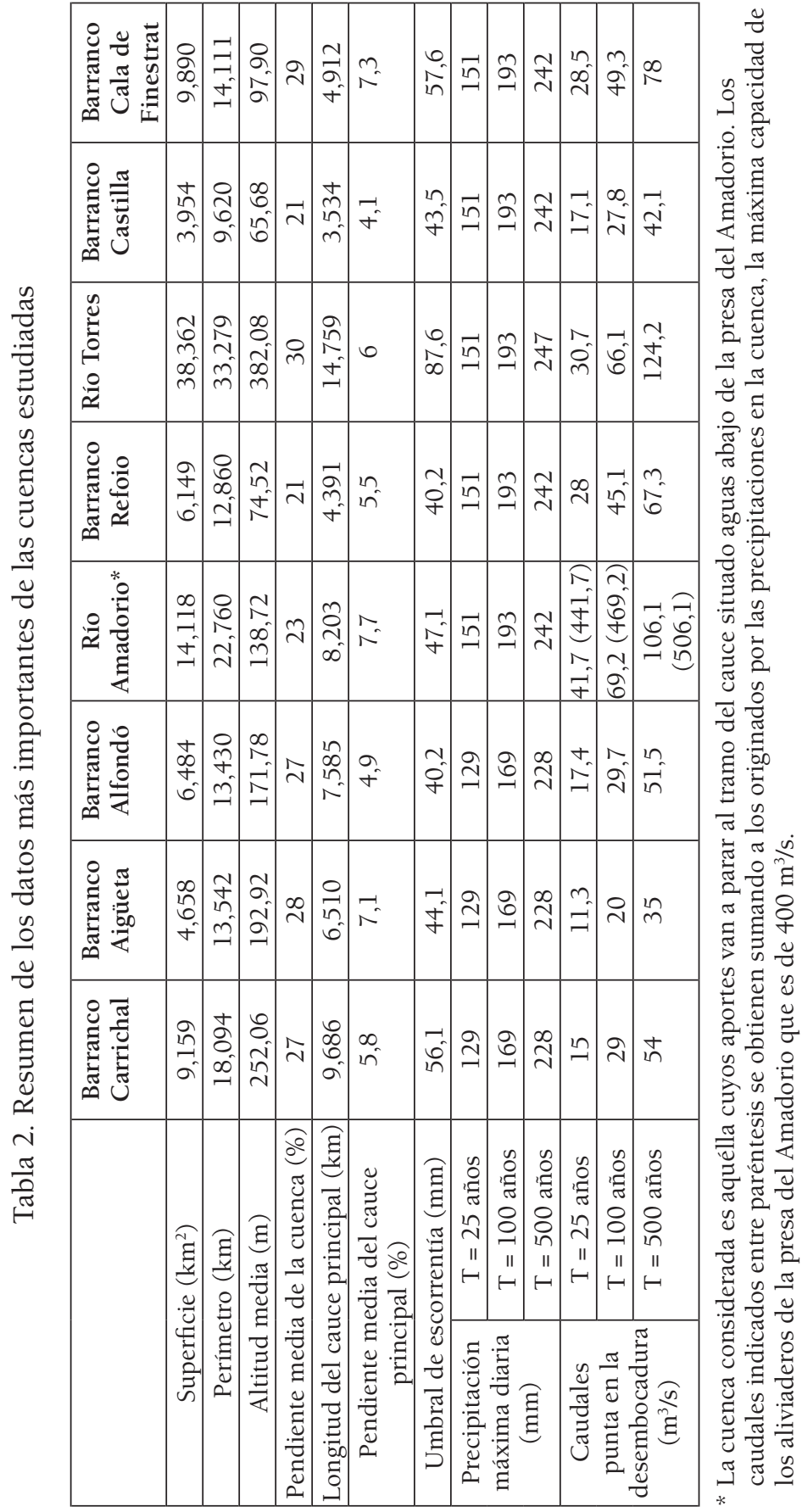


Figura 2. Localización de los barrancos estudiados

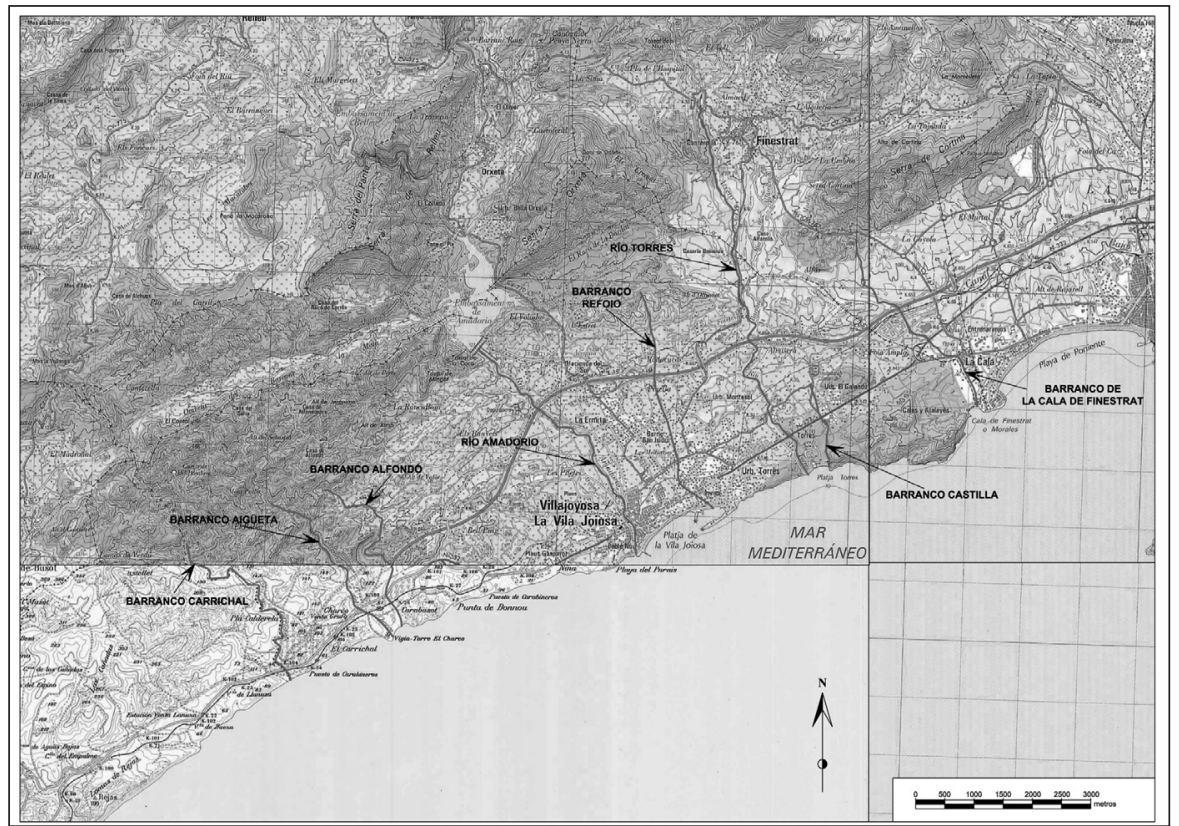

El tramo estudiado de este barranco no se ve afectado por ninguna actuación urbanística según lo indicado por el PGOU de Villajoyosa. Con respecto al PATRICOVA, este barranco no aparece en su listado de zonas en las que efectuar actuaciones para mitigar el riesgo de inundación.

\subsection{Análisis del riesgo de inundación en el tramo final del barranco Aigüeta}

Este barranco nace en la partida Cantalet del término municipal de Villajoyosa, dentro del que se sitúa la totalidad de su cuenca. También se le conoce como barranco del Charco, debido al nombre de la cala donde desemboca.

En el tramo final del barranco, el primer punto de interés es un antiguo azud ubicado a unos centenares de metros aguas arriba de la autopista. Actualmente está catalogado dentro de los bienes protegidos por el ayuntamiento de Villajoyosa y está completamente colmatado. El siguiente punto de interés es la intersección con la autopista AP-7. Ésta se ha resuelto mediante dos canalizaciones prefabricadas de hormigón de sección cuadrada, cuya área es insuficiente para la evacuación de caudales elevados. Este hecho se ve agravado por la existencia de abundante vegetación, que prácticamente tapa las bocas aguas 
arriba de la canalización, obstaculizando aún más la circulación del agua en caso de avenida. Otro detalle de interés en este punto es que se han vertido escombros y tierras (a pesar de la prohibición expresa para ello) en la margen izquierda del cauce, reduciendo su sección de forma considerable.

A $100 \mathrm{~m}$ se encuentra el cruce del barranco con la carretera N-332, que se ha resuelto mediante un puente idéntico al que permitía a dicha carretera salvar el barranco Carrichal. Destaca la presencia de vegetación en su parte inferior, por lo que sería necesaria su limpieza. A priori, es suficiente para permitir el paso de los caudales requeridos. En esta zona, en la margen derecha del barranco (mirando hacia la desembocadura) hay terrazas de cultivo abandonadas que disminuyen su sección. En las proximidades de la desembocadura, el cauce se estrecha y aumenta la presencia de vegetación. Por último, el barranco desemboca en la Cala del Charco, que no presenta ningún problema al ser prácticamente virgen y carecer de ocupaciones.

Con respecto al PGOU de Villajoyosa, este barranco se ve afectado por el plan parcial 31 entre la intersección con la N-332 y su desembocadura. Por la margen izquierda del cauce (mirando aguas abajo) discurrirá un vial, que lo invade de forma parcial. Previsiblemente este vial discurrirá elevado respecto al cauce actual y no se ve afectado por ninguna de las zonas de riesgo definidas en la cartografía. En el PGOU no se detalla la actuación que se realizará en el cauce del barranco. En este estudio se propone integrarlo en el plan parcial mediante un encauzamiento, respetando los límites de inundación definidos por la cartografía de riesgo para el período de retorno de 500 años.

\subsection{Análisis del riesgo de inundación en el tramo final del barranco Alfondó}

Este barranco nace entre las colinas denominadas Alt de Sebastià, Alt de Serena y Alt de Jeroni y desemboca en la Cala del Charco. La totalidad de la cuenca pertenece al término municipal de Villajoyosa. También recibe los nombres de barranco Carabassot y de barranco Salomó.

Por lo general, desde su parte más alta hasta la autopista AP-7 este barranco posee un pequeño cauce, con presencia de vegetación más o menos frondosa según la zona, fundamentalmente en las márgenes. Su intersección con la autopista AP-7 se ha resuelto mediante dos canalizaciones prefabricadas de hormigón de sección cuadrada, cuya área es excesivamente pequeña para la evacuación de elevados caudales. Esto se ve agravado por la existencia de abundante vegetación en sus alrededores y de vertidos de escombros y basuras.

El siguiente punto de interés es el cruce del barranco con el actual trazado de la carretera N-332. Se ha empleado una canalización prefabricada de 
sección circular de gran tamaño que resulta adecuada para la evacuación de los caudales. A continuación, el cauce se intersecta con el antiguo trazado de la carretera $\mathrm{N}-332$, que se emplea como vía de servicio y acceso a los caminos que se dirigen a las playas y casas de campo de las cercanías. Las características del puente son idénticas a las descritas en las intersecciones de esta carretera con los barrancos Carrichal y Aigüeta.

La parte más problemática son los últimos $300 \mathrm{~m}$ antes de la desembocadura, ya que el cauce está invadido por un camino y por una terraza de cultivo abandonada. Además, junto a la terraza a un poco más de altura existen tres casas, cuyos terrenos invaden parcialmente el cauce. Atendiendo a la cartografía de riesgo elaborada, las viviendas no serían afectadas por una avenida, aunque sí que lo serían parte de los terrenos que forman parte de las propiedades. Finalmente, la desembocadura no presenta ningún problema.

Este barranco se ve afectado por los planes parciales 30 y 31 del PGOU de Villajoyosa. La actuación prevista en él es una canalización subterránea, cuya sección no aparece definida, que tendría su origen a unos $150 \mathrm{~m}$ aguas abajo del puente del antiguo trazado de carretera N-332 y que finalizaría en la Cala del Charco. En este estudio se aconseja integrar el cauce en el plan parcial mediante un encauzamiento. Por último, hay que indicar que en el PATRICOVA no se hace mención a este barranco.

\subsection{Análisis del riesgo de inundación en el tramo final del río Amadorio}

Este río desemboca en el núcleo urbano de Villajoyosa y en lo referente a la regulación de su caudal destaca la presencia del pantano del Amadorio. Dada esta circunstancia, para el estudio se ha considerado la parte de la cuenca del río cuyos aportes van a parar al tramo del cauce situado aguas abajo de la presa del Amadorio.

Con respecto al cálculo de caudales necesarios para elaborar la cartografía de riesgo de inundación, se han tenido en cuenta los producidos por la parte de la cuenca indicada más el máximo caudal de desagüe de los aliviaderos de la presa $\left(400 \mathrm{~m}^{3} / \mathrm{s}\right)$. Con ello, se han tratado de simular las condiciones normales más desfavorables que podrían acompañar a la precipitación máxima diaria esperada en los períodos de retorno de 25, 100 y 500 años. No se han contemplado las posibilidades de desbordamiento y rotura de la presa, al ser consideradas situaciones de carácter muy excepcional.

La zona más afectada en caso de avenida es el barrio situado entre la desembocadura y la playa centro de Villajoyosa, conocido como barrio del Arsenal. Además, la situación en este barrio se ve agravada por el hecho de que las 
Figura 3. Cartografía de riesgo de inundación en el tramo final del río Amadorio

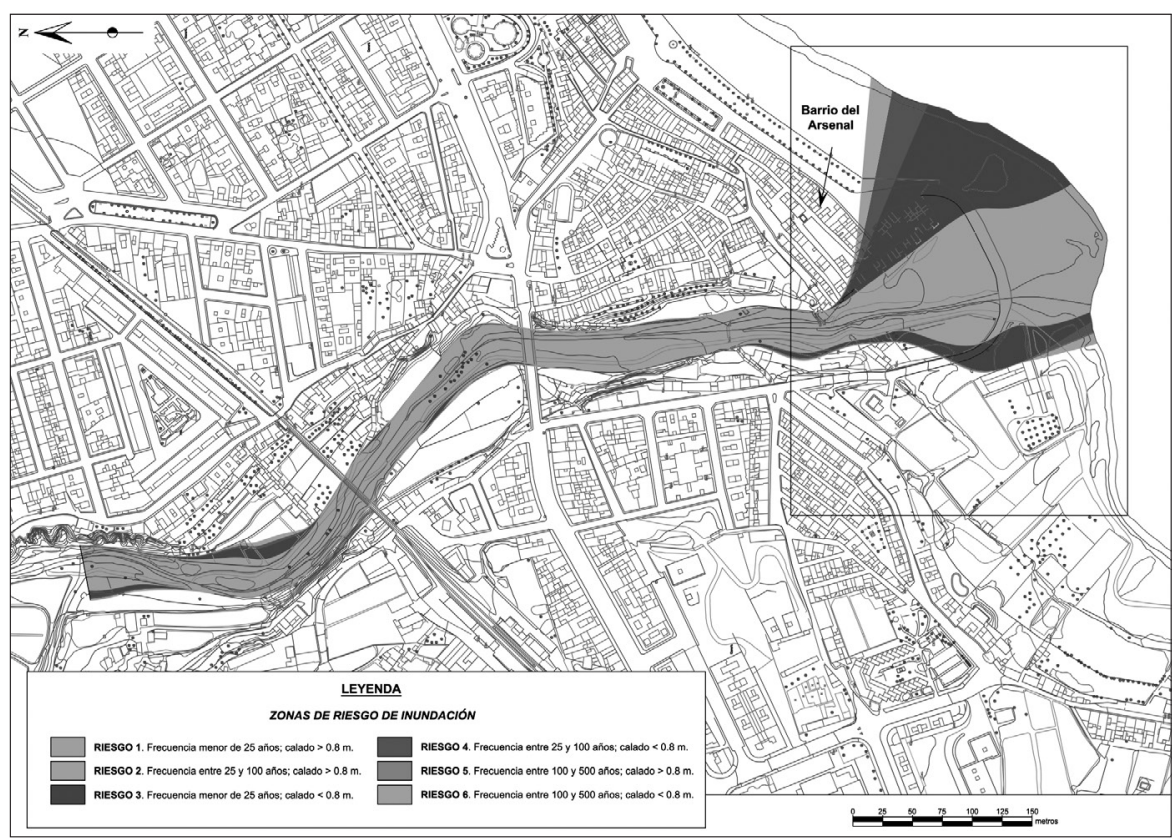

Solamente están presentes los niveles de riesgo 1, 3, 4 y 6, al igual que en todas las cartografías obtenidas como resultado de este estudio. La zona de mayor afección es la desembocadura que aparece resaltada mediante un recuadro.

avenidas del río suelen coincidir con temporales marítimos, produciendo la combinación de ambos unos efectos devastadores.

El tramo urbano del Río Amadorio es el único de este trabajo que aparece dentro de las actuaciones previstas por el PATRICOVA. Concretamente, la actuación se denomina «Encauzamiento del río Amadorio» (código EAC 17). Según lo indicado en su ficha, la descripción de la actuación es adecuar el tramo urbano del río, haciéndolo compatible con los caudales de desagüe del embalse. Los organismos encargados de llevarla a cabo son la Conselleria de Medio Ambiente, Agua, Urbanismo y Vivienda, en colaboración con la Confederación Hidrográfica del Júcar. La prioridad asignada es alta, el tiempo estimado de ejecución es de seis meses con un presupuesto de tres millones de euros. En la actualidad, todavía no se ha realizado ninguna actuación.

Como observación a la actuación prevista, conviene exponer que sólo se exige compatibilizar el cauce con el caudal de desagüe del embalse (hay que recordar que la máxima capacidad de los aliviaderos es de $400 \mathrm{~m}^{3} / \mathrm{s}$ ). Por lo 
Figuras 4 (superior) y 5 (inferior)
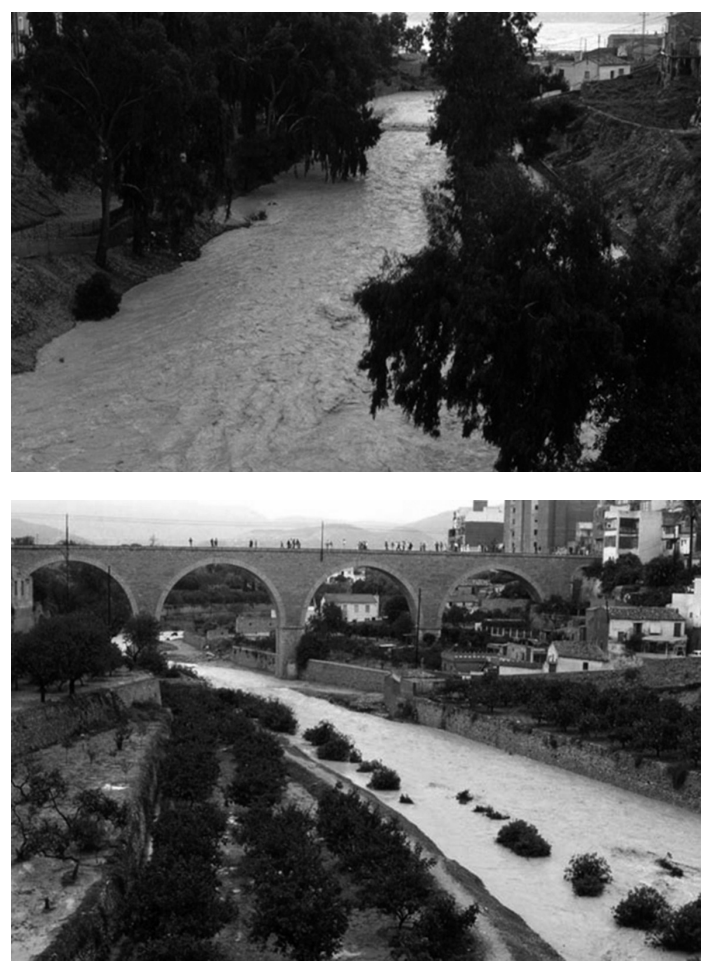

Vistas del tramo urbano del cauce del río Amadorio desde el puente de la carretera $\mathrm{N}-332$, durante la avenida del 4 y 5 de noviembre de 1987. En la figura 4 se observa el cauce desde aguas arriba, con la desembocadura al fondo, mientras que en la figura 5 se ve desde aguas abajo con el puente del ferrocarril Alicante-Denia en un segundo plano.

Autor: Toni Zaragozí (gentileza del Grupo Ecologista Xoriguer).

tanto, no se tienen en cuenta los caudales generados por la cuenca que vierte sus aguas en el tramo de cauce situado aguas abajo de la presa y que según los resultados obtenidos, serían de algo más $100 \mathrm{~m}^{3} / \mathrm{s}$ en la desembocadura para el período de retorno de 500 años.

Además, como se indicará al describir las actuaciones previstas para paliar las inundaciones producidas en la cuenca del barranco Refoio, está previsto a largo plazo desviar sus aguas al cauce del río Amadorio, lo que supondría un incremento de caudal cercano a los $70 \mathrm{~m}^{3} / \mathrm{s}$ en la desembocadura, según las previsiones para el período de retorno de 500 años. Luego, sería aconsejable dimensionar el encauzamiento del tramo urbano del río Amadorio para una capacidad en torno a $600 \mathrm{~m}^{3} / \mathrm{s}$. 


\subsection{Análisis del riesgo de inundación en el tramo final del barranco Refoio}

Esta cuenca vierte sus aguas en el núcleo urbano de Villajoyosa y sus avenidas son las que históricamente más daños han producido en él, junto con las del río Amadorio. En toda la cuenca no existe ningún cauce principal definido, ya que el agua procede de las colinas conocidas como tossals de damunt de l'horta y de la partida Refoios, desplazándose por las áreas deprimidas existentes en la huerta de Villajoyosa, a modo de vaguadas, y acabando en el núcleo urbano.

En el interior de éste no queda ningún vestigio del cauce, ya que el tramo final de la cuenca del Refoio ha quedado completamente invadido de viviendas. Dada esa circunstancia, no se puede aplicar el modelo hidráulico HECRAS. Por ello, la cartografía de riesgo se ha reemplazado por una cartografía de las zonas del núcleo urbano históricamente afectadas por sus avenidas, a modo de conclusión de la recopilación de datos recogidos.

Respecto al análisis de la ocupación antrópica la zona de cabecera de la cuenca, concretamente el pie de las colinas de los tossals de damunt l'horta, se encuentra salpicado de pequeñas edificaciones. Las aguas circulan por los bancales que separan estas viviendas alcanzando así la autopista AP-7, que es atravesada por las principales vaguadas a base de canalizaciones prefabricadas de hormigón. Al entrar en la huerta de Villajoyosa los pequeños cauces se difuminan en depresiones, que constituyen zonas de circulación preferente del agua en caso de avenida. Así se atraviesa la zona de la ermita de San Antonio y la partida de Pinella. Más adelante la cuenca es cruzada por la circunvalación de la carretera N-332 a su paso por Villajoyosa, que a pesar de su reciente construcción actúa como un auténtico dique, con escasas canalizaciones que permitan una mejor permeabilidad de la infraestructura al paso de las aguas de avenida. Únicamente cuenta con algunas canalizaciones prefabricadas de hormigón de pequeña sección, que resultarían insuficientes en caso de avenida. A escasa distancia de la circunvalación se encuentran las primeras edificaciones del núcleo urbano.

Dentro de la trama urbana, el punto donde confluyen prácticamente todas las vaguadas es en la iglesia de El Carmen, ubicada en las antiguas partidas de El Xarquet y Les Barberes actualmente edificadas por completo. Esta iglesia, inicialmente construida en un bajo, fue completamente arrasada en las inundaciones de octubre de 1971, comentando textualmente los testimonios consultados que «los santos salían flotando». Desde allí las aguas transcurren por las calles Marqués de Valterra, Flota Pesquera y Barberes, atravesando el polideportivo de igual nombre. Por último, continúan por una zona de bancales donde destaca la presencia de alguna casa de campo y se dirigen hasta 
Figuras 6 (izquierda) y 7 (derecha)
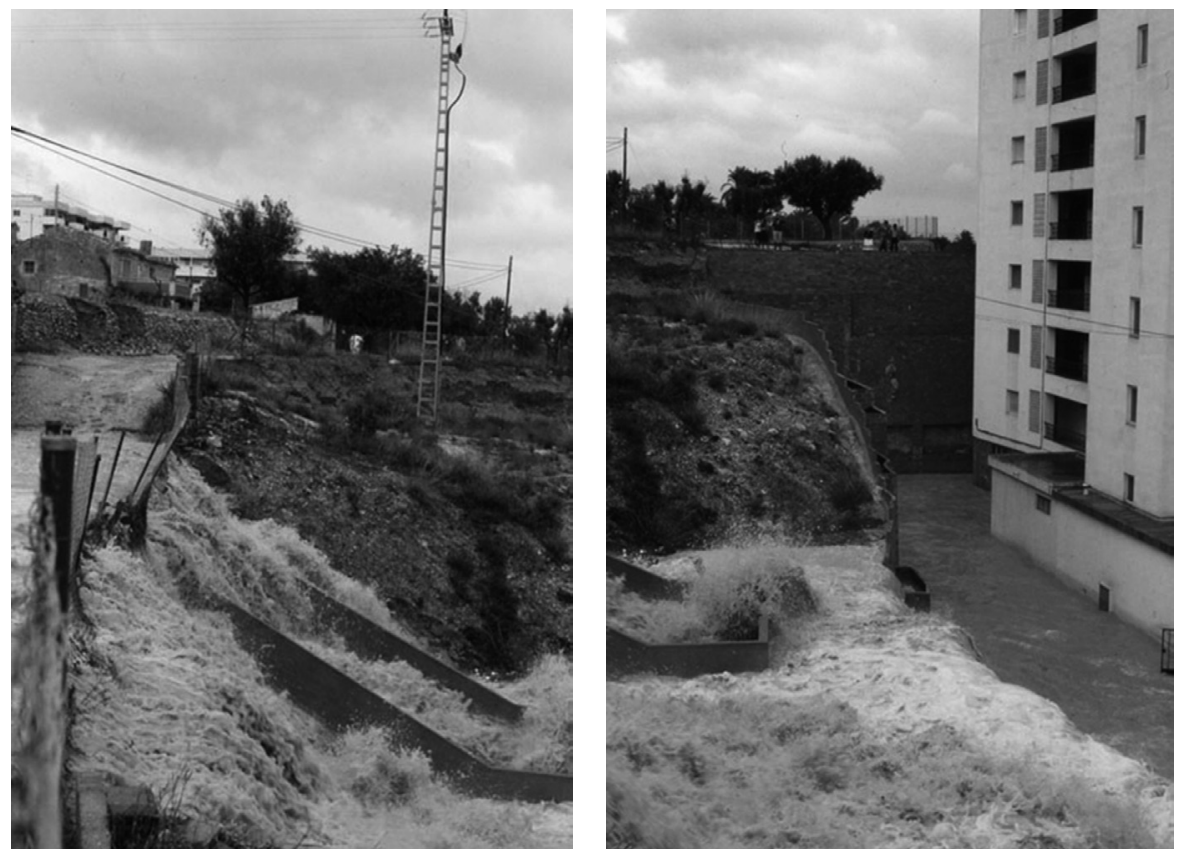

Desembocadura del barranco Refoio en la zona denominada l'esgoladora de l'Almuixó durante las inundaciones del 4 y 5 de noviembre de 1987. En la figura 6 se observa que el colector construido en este punto fue insuficiente para recoger los caudales de la avenida. En la figura 7 destaca la presencia de uno de los edificios que obstaculizan la desembocadura en el actual Club Náutico. Autor: Toni Zaragozí (gentileza del Grupo Ecologista Xoriguer).

la zona conocida como l'esgoladora de l'Almuixó, donde desembocan en el puerto (delante de los muelles del Club Náutico), con un salto de más de 10 $\mathrm{m}$. Curiosamente, en los escasos $150 \mathrm{~m}$ que separan l'esgoladora de l'Almuixó y los muelles, se erigen dos edificios denominados Don Paco y Don Pedro.

En el PGOU la única referencia a esta cuenca se hace a la hora de hablar de la futura vía parque de Villajoyosa. Esta vía todavía no ha empezado a construirse y circunvalará Villajoyosa, atravesando las urbanizaciones recientemente construidas en la periferia de la ciudad. Con el fin de evitar que las aguas de la cuenca del Refoio afecten a las zonas descritas con anterioridad, está previsto construir un colector subterráneo de $2 \mathrm{~m}$ de diámetro junto a la vía parque, para que capte todas las aguas procedentes de esta cuenca y las lleve al río Amadorio. 
Figura 8. Cartografía de zonas afectadas históricamente por las avenidas en la cuenca del barranco Refoio

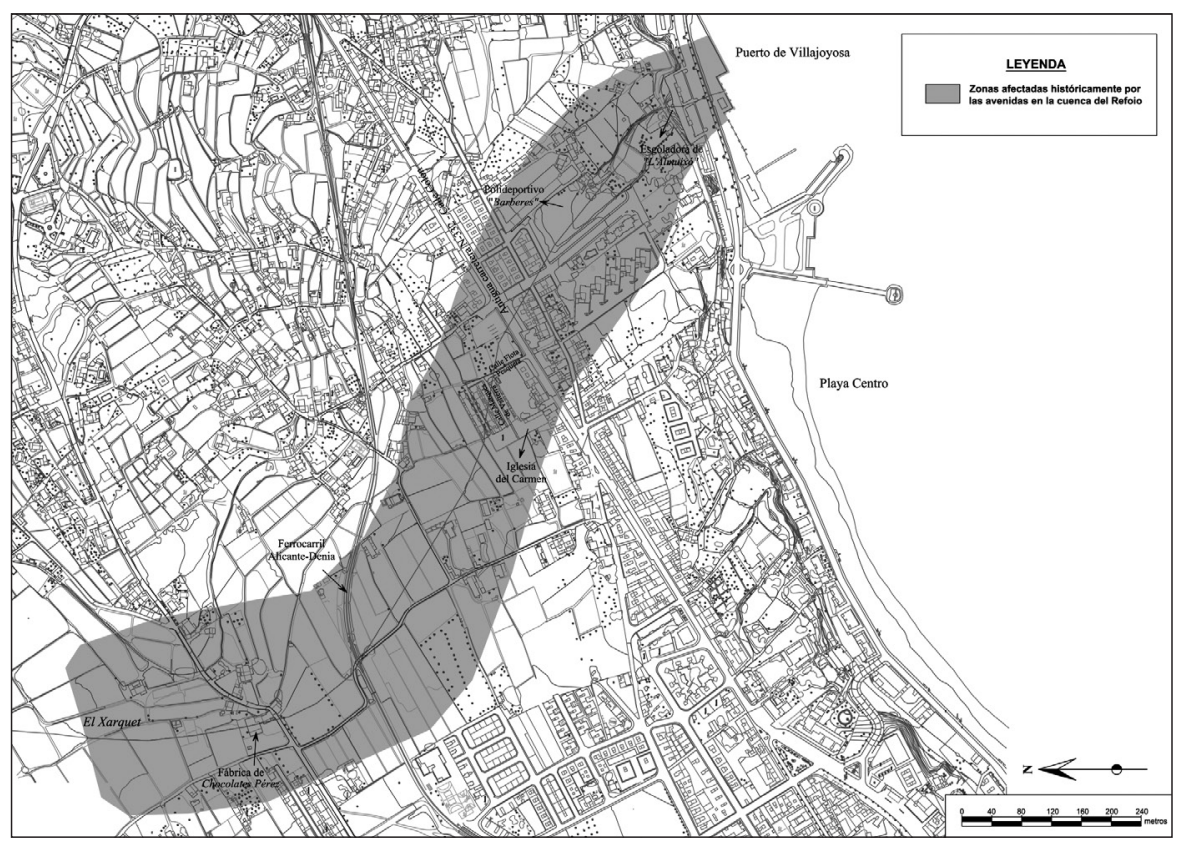

\subsection{Análisis del riesgo de inundación en el tramo final del río Torres}

El río Torres se forma a partir de los barrancos Alginenc, Fondo de Cosme, Murtet, y de los ríos Atxero y Alfarelles. Su cuenca es de gran extensión y su cauce principal nace entre Els Castellets, la umbría del Puig Campana y la sierra de Polop, en la partida conocida como Les Monges. Los términos municipales en los que se sitúa esta cuenca son los de Finestrat y Villajoyosa.

En este caso se han inventariado la ocupaciones del cauce en sus últimos $3,5 \mathrm{~km}$. El primer punto problemático es el puente de la carretera CV-759 que une las localidades de Villajoyosa y Finestrat. Éste se encuentra junto a la confluencia de los ríos Atxero y Alfarelles, dando lugar al que a partir de ese punto se conoce como río Torres. En esta zona el cauce es estrecho y con abundante vegetación, tapando por completo la entrada al puente. Continuando hacia la desembocadura, el río se cruza con la autopista AP-7. La intersección se ha resuelto mediante dos canalizaciones de hormigón prefabricadas de sección semicircular y gran luz, siendo apto para la evacuación de los caudales calculados. Como contrapunto, los principales problemas en caso de avenida los 
causarían los escombros y vegetación acumuladas en su entrada, por lo que sería necesaria una limpieza urgente.

A partir de aquí, las características del cauce se mantienen más o menos constantes, variando su anchura según las zonas y teniendo como denominador común la presencia de abundante vegetación. El siguiente punto de interés es el viaducto de la variante de Villajoyosa de la carretera N-332. Su estructura es a base de pilas y vigas, por lo que no plantea ningún problema para la evacuación de aguas. Sin embargo, durante su construcción se rellenó con tierras gran parte del cauce para permitir el trabajo de maquinaria pesada. Al finalizar las obras estas tierras se retiraron parcialmente, quedando reducida la sección del cauce en los alrededores del viaducto. Por otro lado, a unos centenares de metros del anterior, se halla el puente del antiguo trazado de la carretera N-332 sobre el río. Éste consta de tres luces con sección cada una de ellas en forma un arco de medio punto elevado por hastiales. Es adecuado para los caudales a desaguar, aunque el principal inconveniente es la presencia de vegetación y suciedad en su entorno.

Desde este punto hasta su desembocadura en la playa del Torres, no hay ninguna ocupación destacable. Es digna de mención la abundante vegetación existente que ocupa la totalidad del cauce, con una altura en torno a los $2 \mathrm{~m}$. Un centenar de metros antes de alcanzar la playa, la vegetación pasa a ser en su mayoría a base de matorrales y de baja altura. En este lugar, el cauce está tapado por un relleno de tierra de un metro de altura que sirve de aparcamiento de los coches de los bañistas. Para el paso del agua sólo se han instalado cuatro tubos prefabricados de pequeño diámetro. En la desembocadura, al igual que ocurría con el río Amadorio, hay que tener en cuenta los posibles temporales marítimos, ya que en concomitancia con una avenida los daños aumentarían considerablemente.

En el mes de septiembre de 2007 se cerró la última parte de dos camping que estaban instalados en la playa del Torres. Estos camping se veían afectados frecuentemente tanto por las avenidas del río Torres como del barranco Castilla y por los temporales marítimos. La extensión de terreno que ocupaban era grande y lo más curioso de este caso es que no fueron cerrados por estar en una situación de elevado riesgo de inundación, sino que fue por verse afectados por el plan parcial 27 del PGOU de Villajoyosa. El cierre y desmantelamiento se efectuó por partes, a medida que iban avanzando las obras del citado plan. Con respecto al cauce del río Torres no está prevista ninguna actuación en su tramo final, respetándose teóricamente por parte del plan parcial la zonas de servidumbre y policía correspondientes al dominio público 
hidráulico. A pesar de ello, los movimientos de tierras efectuados en la zona han afectado a una de las márgenes del río.

\subsection{Análisis del riesgo de inundación en el tramo final del barranco Castilla}

Este barranco nace en la solana del monte El Murtet en la partida Foietes d'Horta y desemboca en la Cala del Torres. Discurre en su totalidad por el término municipal de Villajoyosa.

La principal ocupación que ha presentado esta cuenca son los dos camping mencionados al describir el riesgo de inundación en el río Torres, actualmente desmantelados. En caso de avenida, las áreas destinadas al aparcamiento de caravanas y acampada hubiesen quedado completamente inundadas, pudiendo haberse producido muchos daños personales, a parte de los materiales.

Según el PGOU de Villajoyosa el cauce del barranco Castilla se ve afectado en la práctica totalidad del tramo analizado por los planes parciales 26 y 27 . No aparece detallado el tratamiento que tendrá el barranco en los citados

Figura 9. Cartografía de riesgo de inundación en el tramo final del barranco Castilla

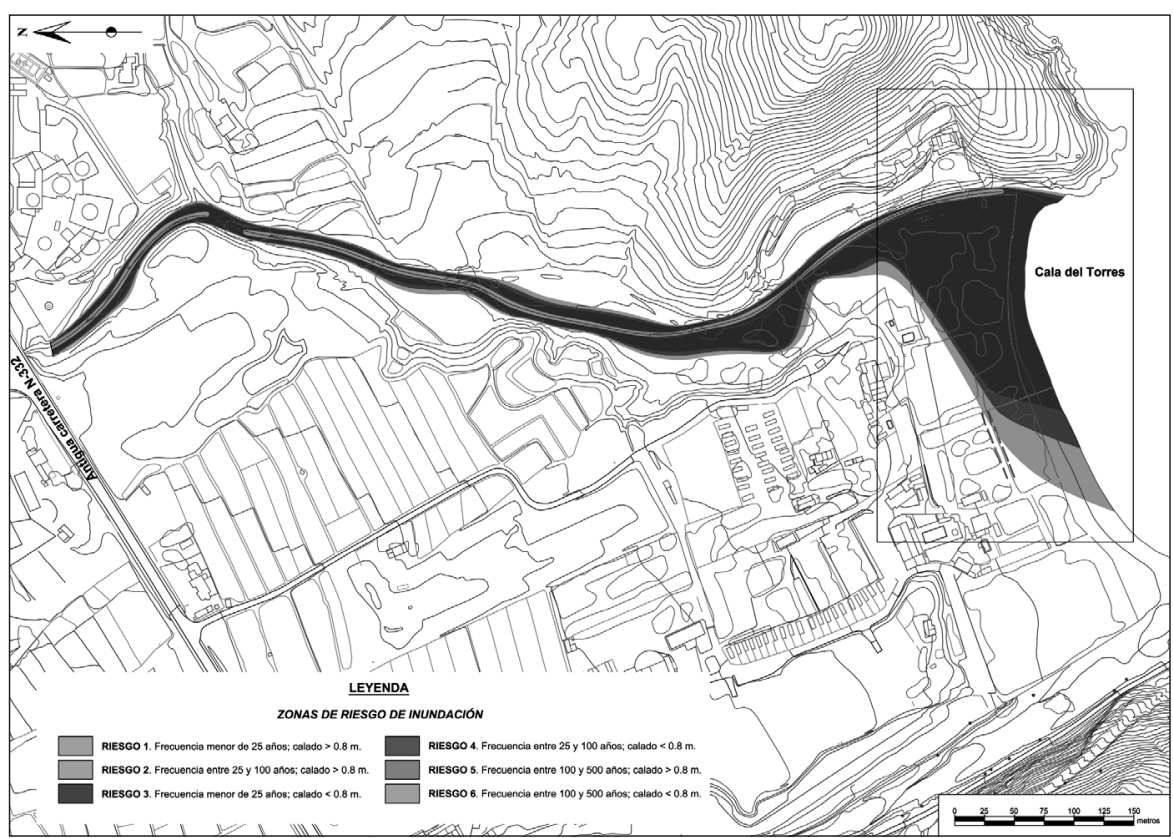

La zona recuadrada corresponde a la Cala del Torres donde se situaban los camping recientemente desmantelados. 
planes parciales, pero de la información extraída del PGOU se entiende que será objeto de una canalización subterránea desde el Casino Mediterráneo hasta su desembocadura en el Cala del Torres. No obstante, lo más adecuado sería realizar un encauzamiento capaz de albergar los caudales esperados para la avenida correspondiente al período de retorno de 500 años. Con ello, se integraría el cauce en los planes parciales anteriores, pudiéndose habilitar como zona verde y recreativa, mejorando el entorno.

\subsection{Análisis del riesgo de inundación en la Cala de Finestrat}

El barranco de la Cala nace en la sierra Cortina y desemboca en la propia Cala de Finestrat. El cauce principal discurre siempre dentro del término municipal de Finestrat, aunque su cuenca se extiende hasta los términos de Villajoyosa y Benidorm.

El barranco de la Cala de Finestrat se encuentra encauzado desde su intersección con el antiguo trazado de la carretera N-332, salvo sus últimos 250 $\mathrm{m}$ donde su continuación natural es la avenida Marina Baixa. En los primeros $550 \mathrm{~m}$ del encauzamiento el lecho es de tierra compactada, mientras que en el resto está asfaltado. En el tramo asfaltado, justo hasta la intersección con la avenida Marina Baixa, todos los viernes se sitúa el mercadillo. Este hecho resulta muy peligroso por la gran aglomeración de gente que suele atraer, no obstante en caso de alerta por fuertes lluvias el mercadillo se suspende. De todos modos, la sensibilidad respecto al riesgo de inundación es importante, como lo hace patente la presencia de bastantes señales recordando el peligro en caso de avenidas.

Junto al tramo encauzado hay edificios de gran altura con parcelas donde hay piscinas, pistas deportivas e instalaciones similares. El principal problema estriba en que estas zonas recreativas están situadas a una cota inferior a la coronación del muro que delimita el encauzamiento, por lo que se inundarían de forma inmediata en caso de desbordarse. Sin embargo, en el análisis del encauzamiento efectuado con HEC-RAS se pone de manifiesto que en líneas generales está bien diseñado, puesto que solamente se detectan posibles desbordamientos en cuatro secciones y siempre en el caso de la avenida correspondiente al período de retorno de 500 años. Para evitar estos posibles desbordamientos sería suficiente con recrecer los muros que delimitan el encauzamiento en torno a un metro o metro y medio.

Por último, en el tramo desde el fin del encauzamiento hasta la desembocadura (avenida Marina Baixa) hay bastantes tiendas y actividad, especialmente en verano. Las tiendas y los bajos de los edificios se verían seriamente afectados en caso de avenida, como se puso de manifiesto en las inundaciones 
Figura 10. Cartografía de riesgo de inundación en el tramo final del barranco de la Cala de Finestrat.

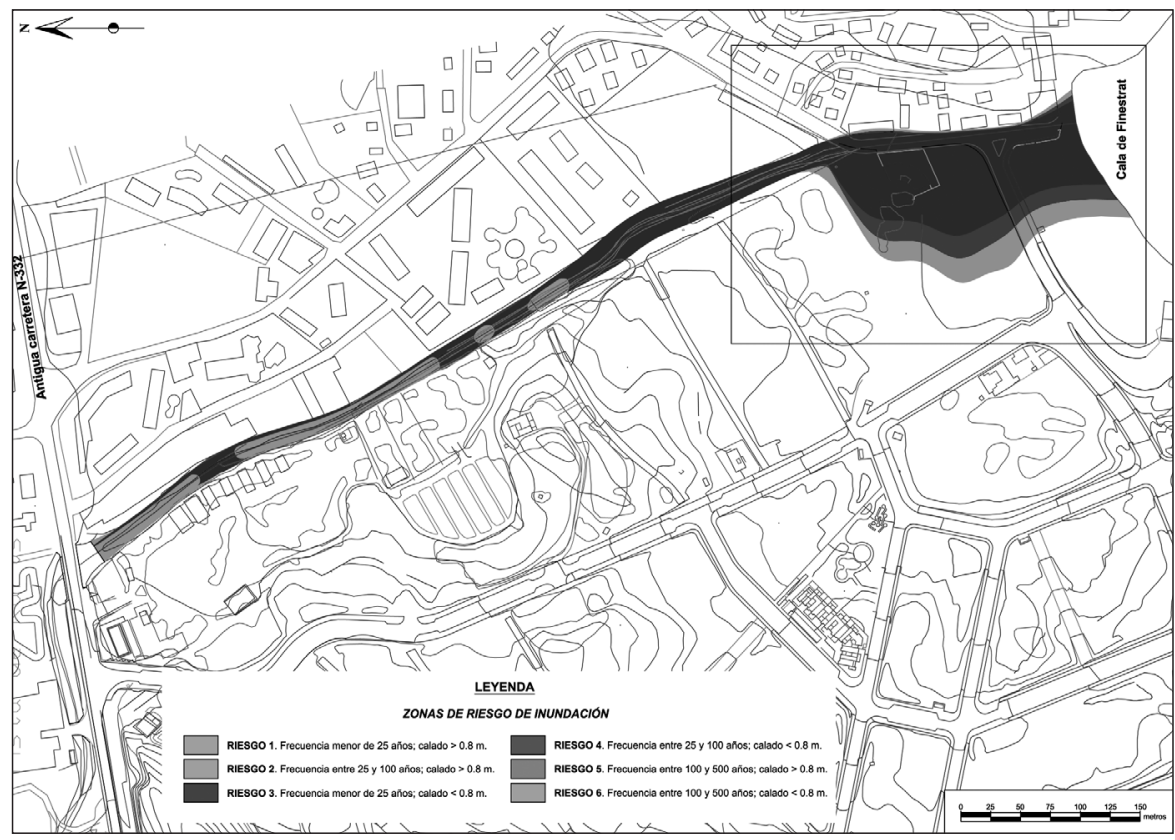

Se nota claramente la parte del tramo correspondiente al encauzamiento y la zona sin encauzar, donde el área afectada en caso de avenida es mucho mayor.

de otoño de 2007. Además, el área de influencia de una avenida tal como se puede ver en la cartografía de riesgo de inundación, no se restringiría únicamente a esta calle, sino que afectaría a las adyacentes, penetrando en el término municipal de Villajoyosa. El problema de este tramo tiene una compleja solución, que se ha agravado al realizar con anterioridad una actuación de dudosa eficacia (el encauzamiento ya realizado).

La solución que se propone en este trabajo es realizar una canalización subterránea del barranco de unos $600 \mathrm{~m}$ aproximadamente de longitud, cuyo punto de inicio sería donde empieza a estar asfaltado el encauzamiento actual y que finalizaría en la playa, penetrando unos metros en el mar. La sección de la canalización ha de dimensionarse para el caudal correspondiente al período de retorno de 500 años. El tramo inicial encauzado, recordemos que estaba sin asfaltar, también habría que modificarlo y darle la pendiente suficiente, para que sirva de transición entre la cota del barranco al salir del puente de la 
carretera N-332 (coincidiría con la actual) y la nueva cota donde se iniciaría el encauzamiento subterráneo.

Finalmente, una vez realizadas las obras, el tramo encauzado que se encontraba asfaltado se dejaría tal como estaba y podría perfectamente emplearse para albergar el mercadillo, tal como se hacía previamente a la ejecución de la canalización subterránea propuesta.

A pesar de esta situación de importante vulnerabilidad, además del encauzamiento ya realizado, no existe ninguna actuación más prevista en un futuro inmediato en la zona estudiada del barranco. Por último, hay que añadir que en el documento PATRICOVA no aparece ninguna referencia respecto a la Cala de Finestrat.

\section{Conclusión}

A lo largo del trabajo efectuado se extrae que la gravedad e importancia del riesgo de inundación en el territorio estudiado varía según la vulnerabilidad de las áreas estudiadas. Esta vulnerabilidad se debe a la presencia de edificios y otro tipo de construcciones sobre antiguos cauces, que en algún caso han desaparecido por completo.

Las zonas que más daños sufrirían en caso de avenida en Villajoyosa son el barrio del Arsenal, adyacente a la desembocadura del río Amadorio, y la zona del Xarquet, partida Barberes y L'Almuixó frente al puerto, debido a las avenidas de la cuenca del barranco Refoio. Las avenidas del río están ligadas en la actualidad a los desagües de la presa del Amadorio, que aunque son muy poco frecuentes, siempre existe la posibilidad de que se produzcan. Las actuaciones previstas en la desembocadura, que incluyen el encauzamiento del río, si se dimensionan correctamente reducirán mucho el riesgo de inundación en esa zona.

Un caso a parte son las inundaciones producidas por la cuenca del barranco Refoio, ya que a causa del crecimiento urbanístico de la localidad en los últimos ocho años y al producirse la última inundación en 1987 es más difícil predecir los lugares de circulación del agua. La propuesta existente de aprovechar la futura vía parque para instalar un colector que desvíe al río las aguas de esta cuenca parece razonable. No obstante, habrá que tenerlo en cuenta para diseñar el encauzamiento del río.

En el resto del término municipal de Villajoyosa, hay que distinguir entre las cuencas que discurren por la parte suroeste del término (barrancos Carrichal, Aigüeta y Alfondó) y las que lo hacen por el noreste (río Torres y barranco Castilla). 
En el primer grupo, el barranco Carrichal no plantea hoy en día ningún problema importante, ya que no está prevista ninguna actuación urbanística en sus alrededores. En cambio, la zona donde se encuentran los barrancos Aigüeta y Alfondó sí que se ve afectada por un plan parcial. Por lo tanto, en esta área todavía se puede compatibilizar la ordenación territorial con el respeto e integración de los cauces naturales, mitigando de ese modo el riesgo de inundación.

En los cauces de la zona noreste ocurre algo similar, especialmente respecto al barranco Castilla, que se ve afectado de lleno por otra actuación urbanística. Además, en esta zona es muy llamativo el hecho de que durante varias décadas dos camping estuvieron instalados en la Cala del Torres, donde desembocan el río homónimo y el barranco Castilla. A lo largo de su existencia y a pesar de haber sido afectados por diferentes episodios de inundaciones, que incluso produjeron víctimas mortales, no fueron desmantelados o trasladados a otra parte. Curiosamente, la actuación urbanística que se lleva a cabo en este sector sí que ha sido capaz de acabar con ellos.

La zona de la Cala de Finestrat es otro ejemplo típico de ocupación de la desembocadura de un cauce. Aquí el problema se ha intentado paliar con el encauzamiento de gran parte del barranco de la Cala a lo largo de la zona ocupada. No obstante, todavía queda por resolver el problema de los últimos $250 \mathrm{~m}$ antes de la desembocadura, cuya solución es compleja.

Para finalizar, hay que resaltar que los valores de los caudales obtenidos (entre 35 y $125 \mathrm{~m}^{3} / \mathrm{s}$ aproximadamente para el período de retorno de 500 años) son relativamente elevados respecto a la pequeña superficie de la mayoría de las cuencas. Esto se debe a la presencia de un sustrato de permeabilidad entre media y baja en las cuencas. Con carácter general, respecto a la metodología es muy importante emplear en estos estudios una topografía de detalle de la zona estudiada (preferiblemente 1:1000 o mayor) y realizar un estudio detallado de las zonas afectadas por inundaciones históricas. Por último, también con carácter universal, la mejor manera de mitigar el riesgo de inundación es compatibilizar las actuaciones urbanísticas con los cauces naturales existentes.

\section{Agradecimientos}

El autor desea agradecer su colaboración en varias de las fases desarrolladas a lo largo de este trabajo a José Miguel Andreu Rodes, Enrique Aparicio Arias, Juan Manuel Gabaldón García, Jorge Olcina Cantos, Carlos Ortega Álvarez, José Payá Nicolau (cronista oficial de Villajoyosa), Alejandro Triviño Pérez y Jaume Vaello Rogles (Grupo Ecologista Xoriguer). 


\section{Bibliografía}

Alfaro, P., J.M. Andreu, A. Estévez, J.E. Tent-Manclús y A. Yébenes (eds.) (2004): Geología de Alicante, autoedición, Alicante, 267 pp.

Agencia Catalana de L'Aigua (2003): Recomanacions tècniques per als estudis d'inundabilitat d'àmbit local, Departament de Medi Ambient de la Generalitat de Catalunya, Barcelona, 89 pp.

Aparicio, F. J. (2003). Fundamentos de hidrología de superficie, Ed. Limusa, Cuernavaca (Méjico), 303 pp.

Departamento de Ciclo Hídrico (2003): Mapa hidrológico provincial de Alicante, Excma. Diputación Provincial de Alicante, Alicante, 48 pp., un mapa.

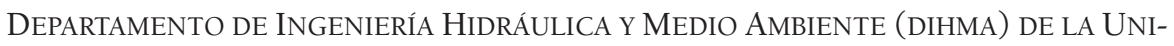
VERSIDAd PolitÉCNICA DE VAlEnCIA (1997): Delimitación del riesgo de inundación en la Comunidad Valenciana, Conselleria d'Obres Publiques, Urbanisme i Transports de la Generalitat Valenciana, Valencia, 72 pp., 1 CD-ROM, 1 plano.

Departamento de Ingeniería Hidráulica y Medio Ambiente (dihma) DE la Universidad Politécnica de VAlencia (2002): Plan de acción territorial de carácter sectorial sobre prevención del riesgo de inundación en la Comunidad Valenciana (PATRICOVA), Conselleria d'Obres Publiques, Urbanisme i Transports de la Generalitat Valenciana, Valencia, 568 pp., 148 planos.

Devesa, M., J. PayÁ y M. LlorCa (2002): Memoria gráfica de La Vila Joiosa, autoedición, Villajoyosa, 258 pp.

DíEz, A., L. LAín y M. LLORENTE (eds.) (2006): Mapas de peligrosidad de avenidas e inundaciones: métodos, experiencias y aplicación, Instituto Geológico y Minero de España, Madrid, 230 pp.

DiRECCIÓN GENERAL DE CARRETERAS (1990): Instrucción 5.2-IC «Drenaje superficial», Ministerio de Obras Públicas y Urbanismo, Madrid, 38 pp.

Dirección General de CARreteras (1999): Máximas lluvias diarias en la España peninsular, Ministerio de Fomento, Madrid, 21 pp., 25 mapas.

GIL, A., J. Olcina y A.M. Rico (editores) (2004): Aguaceros, aguaduchos e inundaciones en áreas urbanas alicantinas, Universidad de Alicante, Alicante, 735 pp.

HERRERO, R. (dir.) (2007): Las zonas inundables de la Comunidad de Madrid: Análisis y cartografía, Dirección General de Urbanismo y Planificación Regional de la Comunidad de Madrid, Madrid, $371 \mathrm{pp}$.

HydROLOGIC ENGINEERING CENTER (2002): HEC-RAS river analysis system: User's manual, version 3.1., Us Army Corps of Engineers, Davis (EE.UU.), 420 pp.

IGME (1981): Mapa geológico de España a escala 1:50000, Instituto Geológico y Minero de España, Madrid, 26 pp., 1 mapa.

Martínez, A. y J. NAVARRo (1996): Hidrología forestal, Universidad de Valladolid, Palencia, 286 pp. 
OlCiNA, J. (1994): Tormentas y granizadas en las tierras alicantinas, Universidad de Alicante, Alicante, 317 pp.

Olcina, J. (1995): Episodios meteorológicos de consecuencias catastróficas en tierras alicantinas (1900-1965), Instituto de Cultura Juan Gil-Albert, Alicante, 376 pp.

PÉREZ, A.J. (coord.) (1994): Atlas climático de la Comunidad Valenciana (19611990), Conselleria d'Obres Públiques, Urbanisme i Transports, Valencia, 205 pp.

SÁNCHEZ, V. (2007): El tiempo que se fue, autoedición, Villajoyosa, 386 pp. 